\title{
CONTAMINATIONS DE CREVETTES ROSES (LEANDER SERRATUS PEN.) AU MOYEN DU CFESIUM I37 PAR VOIE ALIMENTAIRE
}

\author{
par J.C. LEMÉE, J. ANCELLIN et A. VILQUIN * \\ (Manuscrit reçu le 6 inillet 1970)
}

\begin{abstract}
RÉSUMÉ
Des contaminations expérimentales de crevettes roses ont été réalisées par ingestion de moules contaminćes par le cæesium 137 . Une brusque augmentation de la contamination est constatée après chaque repas, suivie d'une décontamination partielle. La contamination résiduelle est évaluée à is \% environ de l'activité de la nourriture et une comparaison établie entre l'importance de la contamination due à l'ingestion et celle intervenant à partir du milieu ambiant montre la prépondérance de cette dernière.
\end{abstract}

\section{SUMMARY}

Shrimps were fed by mussels contaminated with ${ }^{137} \mathrm{Cs}$; a sharp raise of contamination was noticed after each meal followed by partial decontamination. Residual contamination was in the range of is per cent of the diet activity and a comparison between the importance of contamination from either the diet or the environment showed the latter was higher.

\section{I - INTRODUCTION}

La pollution radioactive des mers, provoquée par des retombées d'explosions atomiques et par des rejets d'effluents liquides provenant d'installations nucléaires (usines et navires), a amené le biologiste à prendre conscience du danger potentiel que représente cette pollution. Il convient à ce sujet de s'informer du comportement des radionucléides libérés dans le milieu ambiant, des modes de fixation, des taux de concentration au niveau des organismes, et de faire le relevé des espèces " indicatrices " présentant les pouvoirs de concentration les plus élevés à l'égard d'un radionucléide donné.

Un cas particulier important est celui du transfert de la pollution par l'intermédiaire des chaînes alimentaires qui s'étendent à l'homme. Certaines espèces, en véhiculant les isotopes radioactifs peuvent en effet créer des accumulations secondaires et provoquer la contamination d'espèces géographiquement moins exposées. Il faut donc envisager de suivre le processus de transmission de la contamination d'un niveau trophique à un autre et évaluer les rendements correspondants pour comprendre le rôle des vecteurs de pollution.

* C.E.A. Département de la Protection Sanitaire, Laboratoire de Radioécologie Marine de La Hague, B.P. $n^{\circ} 209$, 50 - Cherbourg.

RADIOPROTECTION, VOL. $6-\mathrm{N}^{0} 2$ 
Nous avons entrepris au Laboratoire de Radioécologie Marine de La Hague, une étude se rapportant à ce dernier aspect des transferts de pollution, en réalisant expérimentalement la contamination secondaire d'une espèce marine, la crevette rose (Leander serratus), par l'intermédiaire de moules (Mytilus edulis) préalablement contaminées par le cæesium-I37 et servant de nourriture.

\section{II - CHOIX DU MATÉRIEL D'ÉTUDE ET DE LA NOURRITURE}

Le choix du matériel d'étude, la crevette rose (Leander serratus), a été dicté par plusieurs raisons :

Io la connaissance des processus de contamination de cette espèce présente un intérêt pratique immédiat puisqu'il s'agit d'un crustacé comestible dont la pêche est active dans le nordCotentin ;

$2^{\circ}$ les crevettes roses sont relativement peu fragiles : leur survie en aquarium fonctionnant en circuit fermé ne présente pas de difficulté et elles se nourrissent facilement ;

$3^{\circ}$ enfin, cette espèce a fait l'objet d'études antérieures au Laboratoire de Radioécologie Marine et nous avons ainsi bénéficié sur place de l'expérience acquise lors de ces travaux [r] [2] [3].

En ce qui concerne la nourriture, le choix a porté sur les moules qu'il était relativement aisé de contaminer en aquarium, et dont on prélevait la chair au fur et à mesure des besoins.

\section{III - CONDITIONS EXPÉRIMENTALES}

\section{io Aquariologie}

a) Pour la contamination des moules servant à l'alimentation, nous avons employé des aquariums en résine armée de fibres de verre $(50 \times 30 \times 40 \mathrm{~cm})$, contenant 40 litres d'eau environ, pourvus de couvercle et fonctionnant en circuit fermé.

b) Les expériences de contamination de crevettes par la seule nourriture exigeaient un milieu ambiant non contaminé. Nous avons utilisé pour chaque crevette un aquarium de petites dimensions contenant 2,5 litres d'eau de mer environ.

Les expériences ont été effectuées dans une salle climatisée en permanence à $14^{-1} 6^{\circ} \mathrm{C}$. L'eau de mer prélevée au voisinage du Centre de La Hague a été introduite non filtrée dans les aquariums.

Les moules servant de nourriture provenaient de la région de Saint-Vaast.

Les crevettes ont été fournies par les pêcheurs locaux.

Les animaux ont été gardés en réserve en aquarium pendant plusieurs jours, avant toute expérimentation, afin d'éliminer les individus les moins résistants.

\section{$2^{\circ}$ RADionUClétDe CONTAMINANT}

Pour les contaminations de moules en aquarium nous avons utilisé le chlorure de cresium-1 37 en solution. Ce radionucléide entre, rappelons-le, dans la composition des rejets radioactifs de l'usine de traitement de combustibles irradiés du Centre de La Hague.

\section{$3^{\circ}$ TeChNiques DE PRÉLÈvements ET DE MESURES}

Des échantillons d'eau de mer ont été régulièrement prélevés dans les aquariums contenant les moules réservées à la nourriture, de manière à suivre l'activité du milieu ambiant contaminant.

Les prélèvements de moules (chair) ont eu lieu suivant les besoins et, avant la mesure, ont été pesés, mais non lavés. De manière à obtenir un coefficient de géométrie relativement constant, chaque prélèvement (eau et moules) occupait un volume de $2 \mathrm{~cm}^{3}$. 
Le but de nos investigations étant de déterminer le transfert de la contamination de la nourriture à l'individu prédateur au cours des repas successifs, nous avons été amenés, d'autre part, à faire la mesure des crevettes vivantes. Cette méthode non seulement écartait certaines difficultés d'interprétation dues aux variations individuelles, mais paraissait s'imposer pour suivre comme il convenait l'évolution de la contamination. Le montage utilisé (fig. 1) assurait une

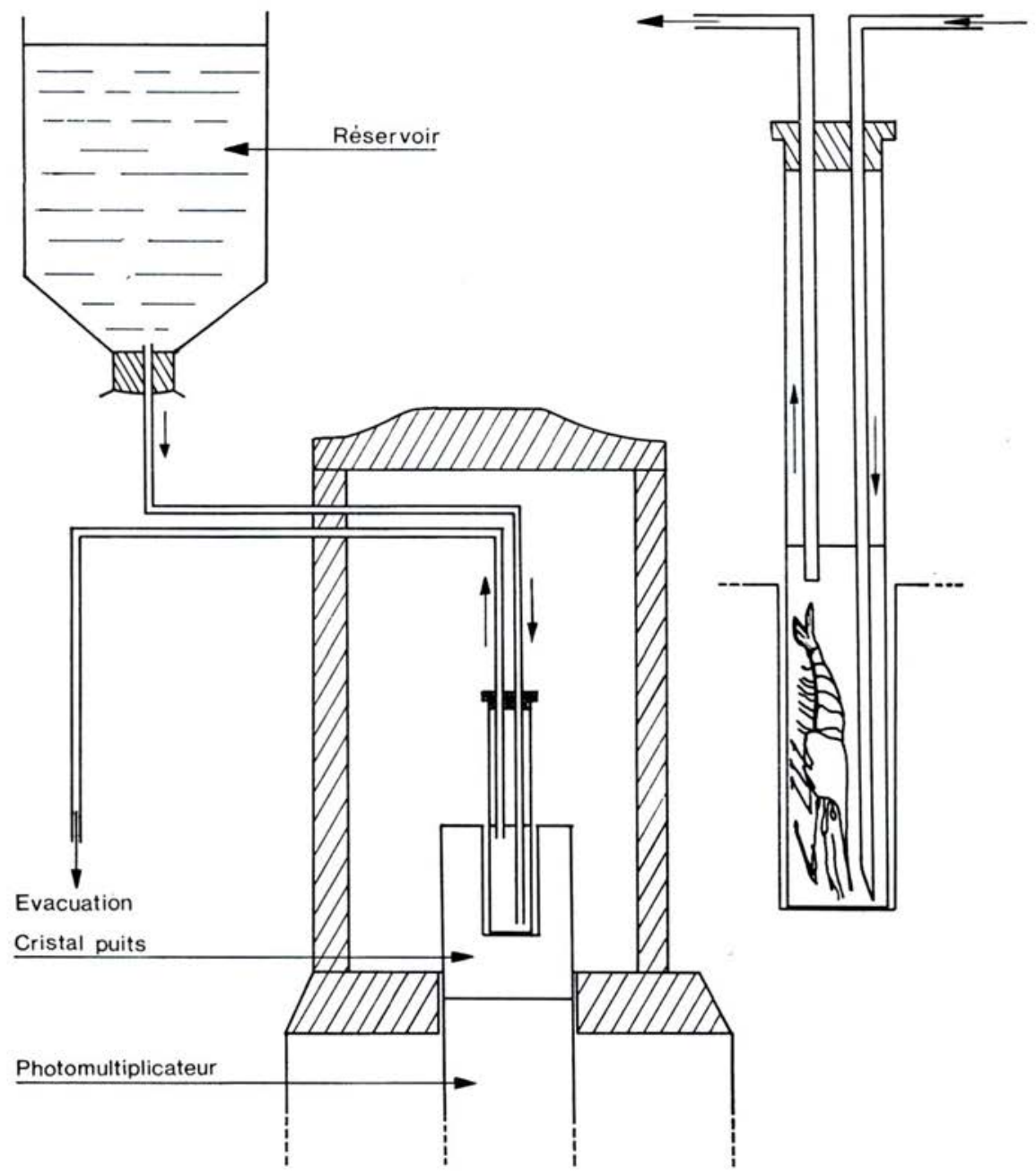

Frg. I. - Montage utilisé pour la mesure d'activité des crevettes vivantes.

circulation permanente d'eau de mer à l'intérieur du tube de comptage et permettait d'éviter une trop importante élévation de la température. Les crevettes ont présenté une très bonne résistance dans ce milieu essentiellement confiné et nous avons pu réaliser des comptages répétés et prolongés sur chaque individu.

vOL. $6-\mathrm{N}^{\circ} 2$ 
Dans tous les cas, les mesures de radioactivité ont été faites par comptages gamma au moyen de détecteurs à scintillation à cristal $\mathrm{INa} \mathrm{I}$ pouce $3 / 4 \times 2$ pouces, à puits standard, dans lequel étaient introduits les tubes de comptage en matière plastique contenant les échantillons à mesurer.

\section{IV - CONTAMINATION DE LA NOURRITURE}

Un lot d'une quarantaine de moules (Mytilus edulis) a été placé dans un aquarium de so litres d'eau de mer contaminée initialement à 4 microcuries par litre. L'activité de la chair (manteau et branchies) a présenté une phase nettement croissante pendant les 20 premiers jours, l'état d'équilibre intervenant vers le $40-50^{e}$ jour. L'activité moyenne de ces tissus correspondait à environ 950 impulsions par minute et par gramme, soit, en valeur absolue, compte tenu du rendement des appareils, environ 4000 à 5000 picocuries par gramme de chair. Le facteur de concentration de l'animal entier par rapport à l'eau était de $12-13$ ( $c f$. tableau I et fig. 2).

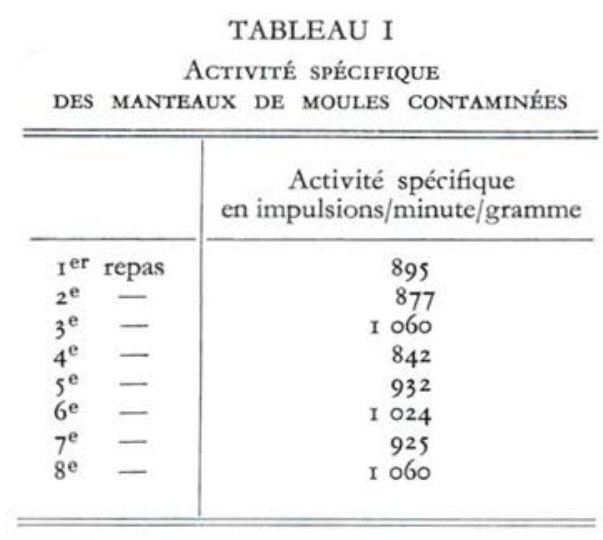

La nourriture des crevettes a été uniquement constituée par des fragments de manteaux et de branchies de moules ayant séjourné environ 3 mois en eau de mer contaminée.

\section{V - CONTAMINATION EXPÉRIMENTALE DES CREVETTES PAR VOIE DIGESTIVE}

Pour réaliser cette étude les crevettes ont été mises, ainsi que nous l'avons déjà indiqué, dans de petits aquariums individuels d'une contenance de 2,5 litres environ. L'eau de mer était fréquemment renouvelée pour éliminer toute contamination du milieu par les excrétions. La nourriture était administrée à raison de deux repas par semaine (fréquence la plus favorable déterminée après étude préalable) régulièrement espacés. L'expérience a porté sur $s$ individus dont les poids étaient les suivants :

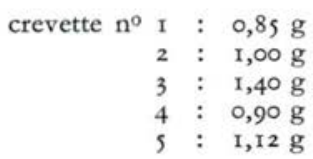

Les manteaux et branchies obtenus par dissection des moules contaminées étaient découpés en fragments, pour faciliter la constitution de la "ration" d'une crevette. Les fragments rassemblés étaient ensuite pesés et comptés globalement pour déterminer l'activité spécifique de la nourriture ainsi préparée. Chaque fragment, enfin, était pesé séparément avant d'être présenté 


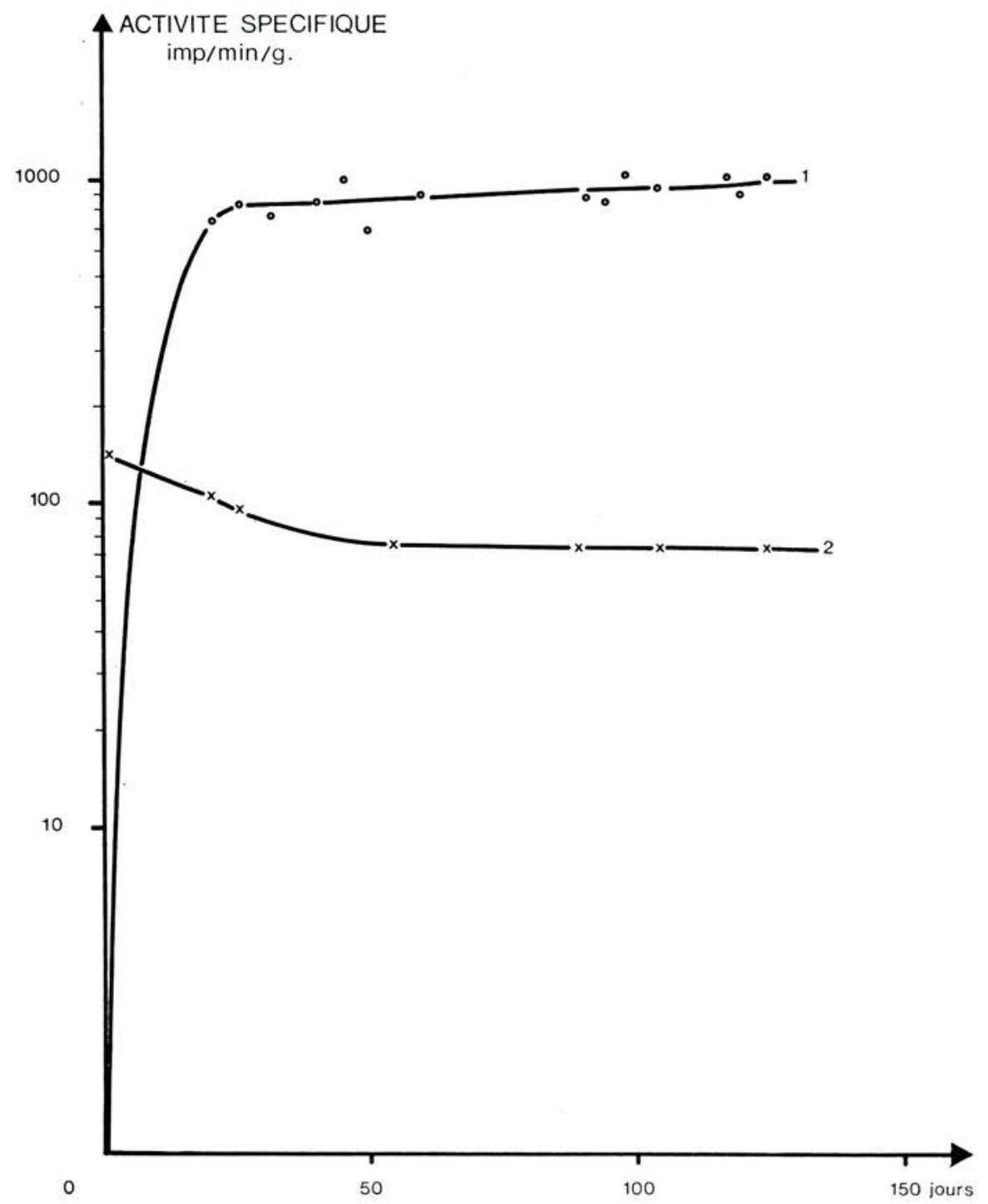

Fig. 2. - Contamination de la nourriture (moules)

courbe $\mathrm{x}$ : activité de la chair,

courbe 2 : activité de l'eau de l'aquarium.

à une crevette déterminée. Ceci permettait dans les cas les plus favorables, où la crevette ingérait en totalité sa "ration", d'apprécier le poids de la nourriture absorbée.

L'expérience a comporté 8 repas consécutifs et a duré 30 jours. Les comptages des individus contaminés par ingestion ont été effectués avant les repas et immédiatement après. Des comp- 
tages intermédiaires permettaient de suivre l'évolution de l'activité de chaque crevette entre les différents repas. On observe (fig. 3 ) une brusque augmentation de la contamination de chaque crevette immédiatement après l'ingestion de la nourriture (section verticale des courbes), puis une décroissance d'activité bien marquée au cours des 48 heures qui suivent chaque repas. Ensuite la décontamination se ralentit et l'on observe une certaine tendance à la stabilisation (paliers).

Cette stabilisation s'établit en définitive à un niveau d'activité relativement faible puisque (sauf pour un individu) il correspond en moyenne à $15-25 \mathrm{imp} . / \mathrm{mn} / \mathrm{g}$.

Nous avons relevé (tableau II), pour chacune des cinq crevettes, les valeurs de l'activité absorbée, d'une part, et de l'activité rejetée, d'autre part, entre deux repas consécutifs, avec indication du " rendement " de chaque repas (\% de l'activité retenue, mesurée immédiatement avant le repas suivant).

\section{TABLEAU II}

Crevette No I

ACTIVITÉ ABSORBÉE, ACTIVITÉ REJETÉE

\begin{tabular}{|c|c|c|c|c|}
\hline Repas & $\begin{array}{c}\text { Activité } \\
\text { absorbée } \\
\mathrm{imp} \cdot / \mathrm{mn} / \mathrm{g}\end{array}$ & $\begin{array}{l}\text { Activité } \\
\text { rejetée } \\
\text { imp./mn/g }\end{array}$ & $\begin{array}{l}\text { Rétention } \\
\mathrm{imp} . / \mathrm{mn} / \mathrm{g}\end{array}$ & $\begin{array}{l}\% \text { d'activité retenue } \\
\text { par rapport } \\
\text { à l'activité absorbée }\end{array}$ \\
\hline I & 30 & 21 & 9 & 30 \\
\hline II & 18 & 17 & I & 6 \\
\hline III & 22 & 19 & 3 & 14 \\
\hline IV & 26 & 21 & 5 & 20 \\
\hline V & 23 & 25 & 0 & $\circ$ / mue \\
\hline VI & 18 & 20 & $\circ$ & of tince \\
\hline Total & I37 & 123 & 14 & $\frac{14}{137}=10 \%$ \\
\hline
\end{tabular}

Activité totale absorbée par la crevette au cours de l'expérience : 550 picocuries de cresium137 (correspondant à $0,12 \mathrm{~g}$ de manteau de moule d'activité spécifique équivalente à $950 \mathrm{imp}$./ $\mathrm{mn} / \mathrm{g}) .90 \%$ ont été rejetés.

Crevette No 2

\begin{tabular}{|c|c|c|c|c|}
\hline Repas & $\begin{array}{c}\text { Activité } \\
\text { absorbée } \\
\mathrm{imp} / \mathrm{mn} / \mathrm{g}\end{array}$ & $\begin{array}{l}\text { Activité } \\
\text { rejetée } \\
\text { imp./mn/g }\end{array}$ & $\begin{array}{l}\text { Rétention } \\
\mathrm{imp} . / \mathrm{mn} / \mathrm{g}\end{array}$ & $\begin{array}{c}\% \\
\text { d'activité } \\
\text { retenue }\end{array}$ \\
\hline I & 30 & 20 & 10 & 33 \\
\hline II & 30 & 25 & 5 & 16 \\
\hline III & 30 & 19 & II & 36 \\
\hline IV & 44 & 39 & 5 & II \\
\hline V & 38 & 28 & Io & 26 \\
\hline VI & $2 \mathrm{I}$ & 24 & 0 & 0 \\
\hline VII & 42 & 55 & 。 & 0 \\
\hline VIII & 17 & 9 & 8 & 45 \\
\hline Total & 252 & 219 & 33 & $\frac{33}{252}=13 \%$ \\
\hline
\end{tabular}

Activité totale absorbée par la crevette au cours de l'expérience : I 200 picocuries de cresium137 (correspondant à $0,26 \mathrm{~g}$ de manteau de moule d'activité spécifique équivalente à $950 \mathrm{imp}$./ $\mathrm{mn} / \mathrm{g}) .87 \%$ ont été rejetés. 
Crevetre $\mathrm{N}^{3} 3$

\begin{tabular}{|c|c|c|c|c|c|}
\hline Repas & $\begin{array}{l}\text { Activité } \\
\text { absorbée } \\
\text { imp./mn/g }\end{array}$ & $\begin{array}{l}\text { Activité } \\
\text { rejetée } \\
\text { imp. } / \mathrm{mn} / \mathrm{g}\end{array}$ & $\begin{array}{l}\text { Rétention } \\
\text { imp. } / \mathrm{mn} / \mathrm{g}\end{array}$ & & $\begin{array}{l}\% \\
\text { d'activité } \\
\text { retenue }\end{array}$ \\
\hline I & 30 & $2 \mathrm{I}$ & 9 & & 30 \\
\hline II & 21 & 18 & 3 & & is \\
\hline III & 42 & 38 & 4 & & Io \\
\hline IV & 39 & 30 & 9 & & 23 \\
\hline V & 17 & 24 & 0 & & o I mu \\
\hline VI & I4 & 18 & $\circ$ & & ○) mue \\
\hline VII & 27 & 25 & 2 & & 7 \\
\hline VIII & 38 & 33 & 5 & & 13 \\
\hline Total & 228 & 207 & 21 & $\frac{2 \mathrm{I}}{228}$ & $9 \%$ \\
\hline
\end{tabular}

Activité totale absorbée par la crevette au cours de l'expérience : I 500 picoruries de casiumI 37 (correspondant à $0,33 \mathrm{~g}$ de manteau de moules d'activité spécifique équivalente à 950 imp./ $\mathrm{mn} / \mathrm{g}) .9 \mathrm{I} \%$ ont été rejetés.

Crevette No 4

\begin{tabular}{|c|c|c|c|c|}
\hline Repas & $\begin{array}{c}\text { Activité } \\
\text { absorbée } \\
\text { imp./mn/g }\end{array}$ & $\begin{array}{l}\text { Activité } \\
\text { rejetée } \\
\mathrm{imp} / \mathrm{mn} / \mathrm{g}\end{array}$ & $\begin{array}{l}\text { Rétention } \\
\text { imp. } / \mathrm{mn} / \mathrm{g}\end{array}$ & $\begin{array}{c}\% \\
\text { d'activité } \\
\text { retenue }\end{array}$ \\
\hline I & 25 & 17 & 8 & 30 \\
\hline II & 19 & 16 & 3 & 16 \\
\hline III & I I & I I & 0 & 0 \\
\hline IV & 40 & 30 & 10 & 25 \\
\hline Total & 95 & 74 & $2 I$ & $\frac{21}{95}=22 \%$ \\
\hline
\end{tabular}

Activité totale absorbée par la crevette au cours de l'expérience : 400 picocuries de cæsium137 (correspondant à $0,10 \mathrm{~g}$ de manteau de moules d'activité spécifique équivalente à 950 imp./ $\mathrm{mn} / \mathrm{g}) .78 \%$ ont été rejetés.

Crevetre Nos

\begin{tabular}{|c|c|c|c|c|c|}
\hline Repas & $\begin{array}{l}\text { Activité } \\
\text { absorbée } \\
\text { imp./mn/g }\end{array}$ & $\begin{array}{l}\text { Activité } \\
\text { rejetée } \\
\text { imp./mn/g }\end{array}$ & $\begin{array}{l}\text { Rétention } \\
\text { imp. } / \mathrm{mn} / \mathrm{g}\end{array}$ & & $\begin{array}{c}\% \\
\text { d'activité } \\
\text { retenue }\end{array}$ \\
\hline I & 30 & I 8 & 12 & & 40 \\
\hline II & 37 & 30 & 7 & & 17 \\
\hline III & 2 I & 20 & I & & 5 \\
\hline IV & 14 & 16 & 0 & & 0 \\
\hline V & $2 I$ & I 8 & 3 & & is \\
\hline Total & 123 & 102 & $2 \mathrm{I}$ & $\frac{21}{123}$ & $=17 \%$ \\
\hline
\end{tabular}

Activité totale absorbée par la crevette au cours de l'expérience : 650 picocuries de cæsium137 (correspondant à $0,15 \mathrm{~g}$ de manteau de moules d'activité spécifique équivalente à 950 imp./ $\mathrm{mn} / \mathrm{g}) .83 \%$ ont été rejetés.

vol. $6-\mathrm{N}^{\circ} 2$ 
I 40

J.C. LEMÉE, J. ANCELLIN ET A. VILQUIN

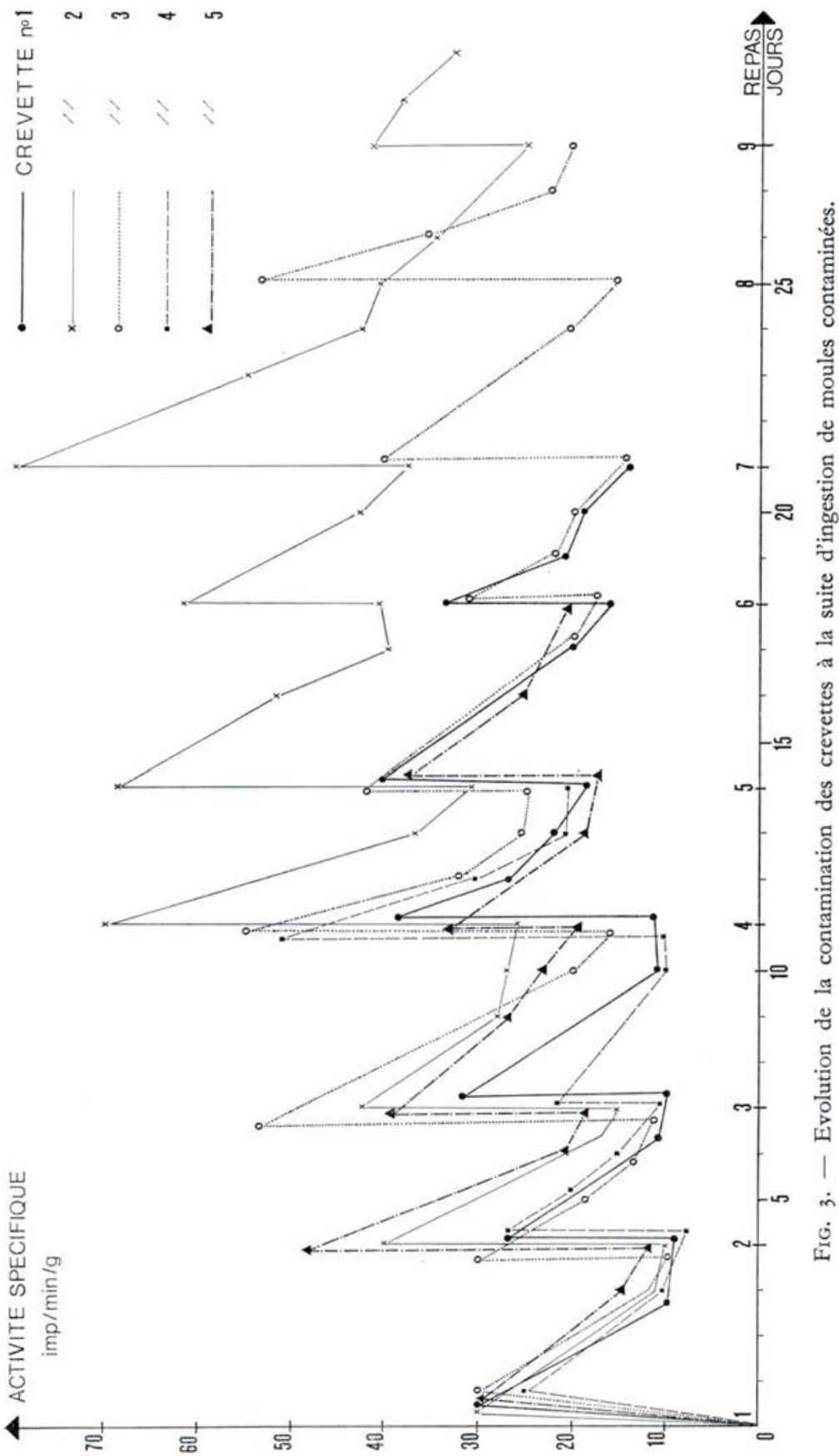

RADIOPROTECTION 
Les résultats de ces expériences permettent de constater que l'ingestion de moules contaminées entraîne chez les crevettes une contamination résiduelle représentant environ ro à $15 \%$ de l'activité absorbée. En période de mue, alors que la crevette mange peu ou pas, la décontamination continue à se manifester, avec diminution corrélative du pourcentage de l'activité retenue. Cependant il convient d'observer que l'augmentation d'activité spécifique de ces crustacés à la suite d'ingestion de moules contaminées est demeurée très inférieure au niveau qui, théoriquement, aurait dû être atteint : les individus d'environ I gramme que nous avons utilisés consomment en effet facilement le dixième de leur poids en un seul repas. Or l'ingestion de $\circ, \mathrm{I} \mathrm{g}$ de chair d'une activité spécifique correspondant à $950 \mathrm{imp} . / \mathrm{mn} / \mathrm{g}$ devrait déterminer chez l'individu une augmentation d'activité spécifique équivalente à $95 \mathrm{imp} . / \mathrm{mn} / \mathrm{g}$, alors que les valeurs constatées demeurent dans la plupart des cas inférieures à $50 \mathrm{imp} . / \mathrm{mn} / \mathrm{g}$.

Nous avons été amenés à supposer qu'une certaine décontamination de la nourriture se produit au cours meme du repas. Nous avons tout d'abord pu nous assurer que les fragments de manteau de moules ne se décontaminaient pas de façon sensible par la seule immersion dans de l'eau de mer inactive. Par contre, la comparaison entre l'augmentation d'activité consécutive à un repas et la mesure précise du poids de manteau de moule ingéré (obtenu dans le cas où la crevette dévorait entièrement sa ration soigneusement pesée au préalable) permettait de constater que la perte d'activité de la nourriture atteignait facilement dans ce cas $50 \%$ de la valeur initiale (tableau III).

\section{TABLEAU III}

\section{DÉCONTAMINATION DE LA NOURRITURE}

\begin{tabular}{|c|c|c|c|c|}
\hline $\begin{array}{c}\text { Crevettes } \\
n^{\circ}\end{array}$ & $\begin{array}{c}\text { Activité absorbée } \\
\text { par repas } \\
\text { (en imp./mn/g) }\end{array}$ & $\begin{array}{c}\text { Equivalence } \\
\text { théorique } \\
\text { de l'activité absorbée } \\
\text { en poids de chair }\end{array}$ & $\begin{array}{l}\text { Poids de chair } \\
\text { effectivement } \\
\text { consommé }\end{array}$ & $\begin{array}{l}\text { Estimation en \% } \\
\text { de la } \\
\text { "décontamination" } \\
\text { de la nourriture }\end{array}$ \\
\hline I & 22 & $0,025 \mathrm{~g}$ & $0,070 \mathrm{~g}$ & 64 \\
\hline 2 & $\begin{array}{l}29 \\
44 \\
38\end{array}$ & $\begin{array}{ll}0,030 & \mathrm{~g} \\
0,050 & \mathrm{~g} \\
0,040 \mathrm{~g}\end{array}$ & $\begin{array}{l}0,070 \mathrm{~g} \\
0,090 \mathrm{~g} \\
0,070 \mathrm{~g}\end{array}$ & $\begin{array}{l}58 \\
45 \\
43\end{array}$ \\
\hline 3 & $\begin{array}{l}28 \\
43 \\
50\end{array}$ & $\begin{array}{ll}0,030 & \mathrm{~g} \\
0,050 & \mathrm{~g} \\
0,052 & \mathrm{~g}\end{array}$ & $\begin{array}{ll}0,100 & g \\
0,085 & g \\
0,150 & g\end{array}$ & $\begin{array}{l}70 \\
42 \\
66\end{array}$ \\
\hline 4 & 33 & $0,035 \mathrm{~g}$ & $0,080 \mathrm{~g}$ & 57 \\
\hline
\end{tabular}

Nous pensons que cette décontamination résulte de la dilacération de la nourriture par les pièces buccales de la crevette dans l'eau ambiante non contaminée.

Il y a lieu de tenir compte de cette dernière constatation en ce qui concerne l'évolution de la contamination résiduelle des crevettes. Si l'on exprime, comme nous l'avons précédemment fait, l'activité résiduelle des crevettes en fonction de la quantité réellement ingérée, le taux de rétention est de is \% environ. Si l'on estime que l'activité de la nourriture préparée subit une décontamination de $50 \%$ par suite de la dilacération précédant l'ingestion, les crevettes n'ont finalement retenu que is \% des so \% de l'activité résiduelle de la nourriture, soit environ $7 \%$ de l'activité initiale (avant dilacération de la nourriture).

Afin d'avoir un aperçu de la répartition du radioélément dans l'organisme, nous nous sommes livrés à une dissection sommaire des crevettes sacrifiées en fin d'expérience ou mortes prématurément. Nous avons pu constater que la presque totalité de l'activité se retrouvait dans le muscle caudal et le tube digestif. Les carapaces obtenues par dissection ou récupérées au cours des mues n'ont pas présenté d'activité décelable.

Ces données sont à comparer avec les résultats de contamination par casium-137, à partir du milieu ambiant (eau de mer) et, pour la même espèce, obtenus au Laboratoire de Radioécologie

vol. $6-\mathrm{N}^{\mathrm{O}} 2$ 
Marine de La Hague. Nous rappellerons brièvement qu'en aquarium fonctionnant en circuit fermé et pour une contamination initiale de $\mathrm{r}$ à $\mathrm{x}, 3$ microcurie par litre d'eau de mer, l'activité spécifique des crevettes alimentées avec une nourriture non contaminée montre d'abord une augmentation régulière puis se stabilise vers le 30 ou $40^{\mathrm{e}}$ jour.

Les facteurs de concentration, par rapport à l'eau de mer, se situent vers $30-40$ au moment où l'équilibre est atteint [r].

Il apparait que la contamination à partir du milieu ambiant dont l'activité spécifique est sensiblement plus faible que celle de la nourriture (de l'ordre de $250 \mathrm{pCi} / \mathrm{g}$ en phase d'équilibre alors que la nourriture est 20 fois plus contaminée) détermine dans l'organisme une activité de $7500 \mathrm{pCi} / \mathrm{g}$ environ, tandis que la consommation de la chair contaminée détermine une activité résiduelle de so à roo $\mathrm{pCi} / \mathrm{g}$ environ.

Ces constatations rejoignent celles déjà présentées par BRYAN et WARD [4] concernant l'importance comparée de ces deux modes de contamination.

\section{VI - CONCLUSION}

- La contamination de crevettes au moyen de cxsium-137, par l'intermédiaire d'une nourriture (chair de moule) préalablement contaminée avec ce radionucléide se traduit, à la suite de chacun des repas, par une brusque augmentation de la radioactivité des individus, suivie d'une décontamination partielle. L'activité résiduelle constatée après plusieurs repas représente environ $15 \%$ de l'activité réellement absorbée par voie alimentaire.

- L'importance de la contamination par voie alimentaire paraît relativement minime par rapport à celle provoquée par l'eau ambiante contaminée, même si l'activité spécifique de celle-ci est faible au regard de l'activité spécifique de la nourriture.

\section{BIBLIOGRAPHIE}

[1] Ancellin J., Michon G. et Vilquin A. Contaminations expérimentales de crevettes roses par le cresium-137. Rapport CEA R. 2818, 1965.

[2] AnCELlin J. et VILQuin A. Contaminations expérimentales d'espèces marines par le cérium 144, le ruthénium ro6 et le zirconium 95. Disp. of radioactiv. wastes into seas, oceans and surface waters. A.I.E.A., Vienne, pp. $583-604,1966$.

[3] Ancellin J. et Vilquin A. Nouvelles contaminations expérimentales d'espèces marines par le cresium-137, le ruthénium I06, et le cérium i44. Radioprotection, vol. $3, \mathrm{n}^{\circ} 3$, pp. $185-213,1968$.

[4] BRyan G.W. et Ward E. Potassium metabolism and the accumulation of 137 cresium by decapod crustacea. J.M.B.A. of the U.K., vol. 42 , n ${ }^{\circ}$, pp. 199. $24 \mathrm{I}, 1962$. 